\title{
HANDBOOK OF PI AND PID CONTROLLER TUNING RULES
}

3rd Edition

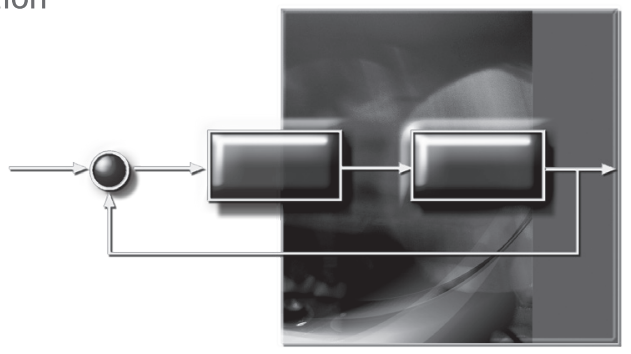


This page intentionally left blank 


\title{
HANDBOOK OF PI AND PID CONTROLLER TUNING RULES
}

\author{
3rd Edition
}

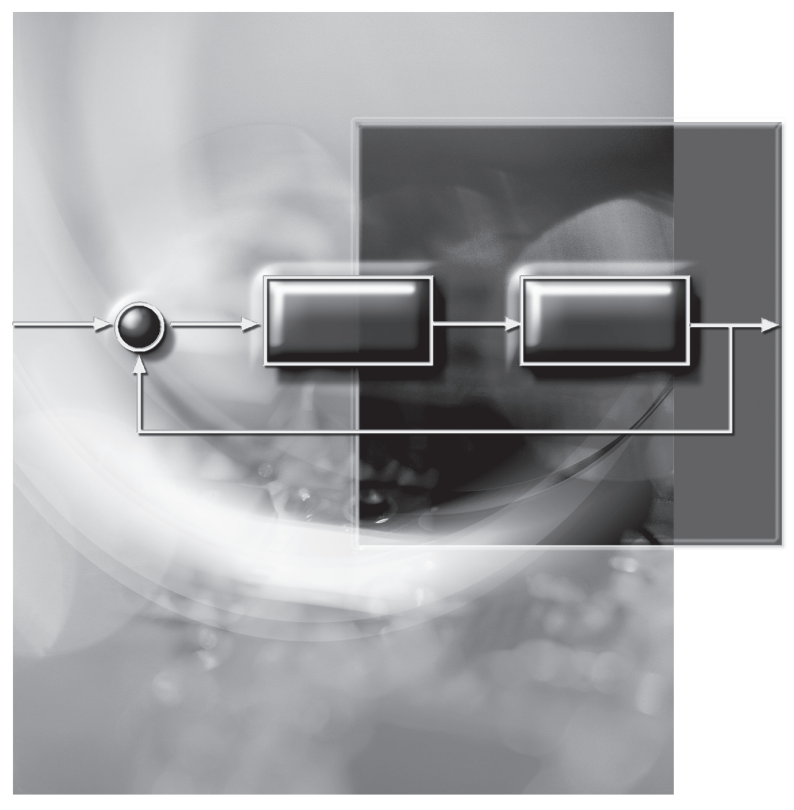

Aidan O'Dwyer

Dublin Institute of Technology, Ireland 
Published by

Imperial College Press

57 Shelton Street

Covent Garden

London WC2H 9HE

\section{Distributed by}

World Scientific Publishing Co. Pte. Ltd.

5 Toh Tuck Link, Singapore 596224

USA office: 27 Warren Street, Suite 401-402, Hackensack, NJ 07601

UK office: 57 Shelton Street, Covent Garden, London WC2H 9HE

\section{British Library Cataloguing-in-Publication Data}

A catalogue record for this book is available from the British Library.

\section{HANDBOOK OF PI AND PID CONTROLLER TUNING RULES (3rd Edition)}

Copyright @ 2009 by Imperial College Press

All rights reserved. This book, or parts thereof, may not be reproduced in any form or by any means, electronic or mechanical, including photocopying, recording or any information storage and retrieval system now known or to be invented, without written permission from the Publisher.

For photocopying of material in this volume, please pay a copying fee through the Copyright Clearance Center, Inc., 222 Rosewood Drive, Danvers, MA 01923, USA. In this case permission to photocopy is not required from the publisher.

ISBN-13 978-1-84816-242-6

ISBN-10 1-84816-242-1

Typeset by Stallion Press

Email: enquiries@stallionpress.com

Printed in Singapore. 


\section{Dedication}

Once more, this book is dedicated with love to Angela, Catherine and Fiona and to my parents, Sean and Lillian. 
This page intentionally left blank 


\section{Preface}

Proportional integral (PI) and proportional integral derivative (PID) controllers have been at the heart of control engineering practice for over seven decades. However, in spite of this, the PID controller has not received much attention from the academic research community until the past two decades, when work by K.J. Åström, T. Hägglund and F.G. Shinskey, among others, sparked a revival of interest in the use of this 'workhorse' of controller implementation.

There is strong evidence that PI and PID controllers remain poorly understood and, in particular, poorly tuned in many applications. It is clear that the many controller tuning rules proposed in the literature are not having an impact on industrial practice. One reason is that the tuning rules are not accessible, being scattered throughout the control literature; in addition, the notation used is not unified. The purpose of this book, now in its third edition, is to bring together and summarise, using a unified notation, tuning rules for PI and PID controllers. The author restricts the work to tuning rules that may be applied to the control of processes with time delays (dead times); in practice, this is not a significant restriction, as most process models have a time delay term. In this edition, the structure of the book has been modified from the previous edition, with controller tuning rules for non-self-regulating process models being summarised in a different chapter from those for self-regulating process models.

It is the author's belief that this book will be useful to control and instrument engineering practitioners and will be a useful reference for students and educators in universities and technical colleges.

I wish to thank the School of Electrical Engineering Systems, Dublin Institute of Technology, for providing the facilities needed to complete the book. Finally, I am deeply grateful to my mother, Lillian, and my father, Sean, for their inspiration and support over many years and to my family, Angela, Catherine and Fiona, for their love and understanding. 
This page intentionally left blank 


\section{Contents}

Preface

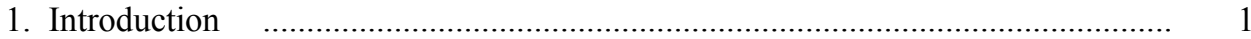

1.1 Preliminary Remarks ........................................................................... 1

1.2 Structure of the Book............................................................................... 2

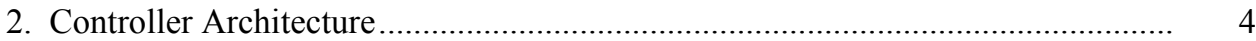

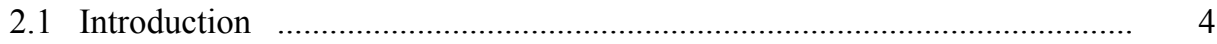

2.2 Comments on the PID Controller Structures . ............................................. 11

2.3 Process Modelling ................................................................................. 12

2.3.1 Self-regulating process models ...................................................... 12

2.3.2 Non-self-regulating process models.............................................. 14

2.4 Organisation of the Tuning Rules ........................................................... 16

3. Controller Tuning Rules for Self-Regulating Process Models ............................. 18

3.1 Delay Model ..................................................................................... 18

3.1.1 Ideal PI controller - Table 2 ...................................................... 18

3.1.2 Ideal PID controller - Table 3 ................................................. 23

3.1.3 Ideal controller in series with a first order lag - Table 4 ............... 24

3.1.4 Classical controller - Table 5 ..................................................... 25

3.1.5 Generalised classical controller - Table 6 ................................... 26

3.1.6 Two degree of freedom controller 1 - Table 7 ................................ 27

3.2 Delay Model with a Zero ......................................................................... 28

3.2.1 Ideal PI controller - Table 8 ...................................................... 28

3.3 FOLPD Model .................................................................................... $\quad 30$

3.3.1 Ideal PI controller - Table 9 ....................................................... $\quad 30$

3.3.2 Ideal PID controller - Table 10 .................................................... 78

3.3.3 Ideal controller in series with a first order lag - Table $11 \ldots \ldots \ldots \ldots \ldots . . .118$

3.3.4 Controller with filtered derivative - Table 12............................... 122

3.3.5 Classical controller - Table 13 ....................................................... 134

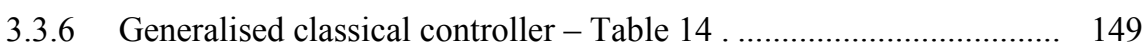

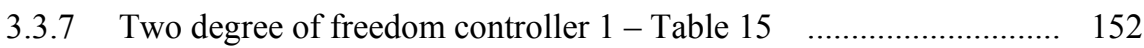

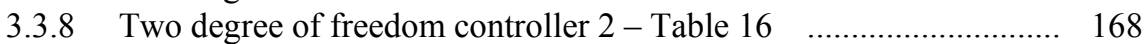

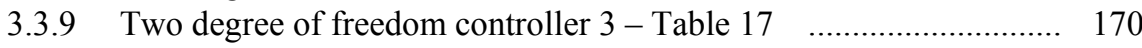




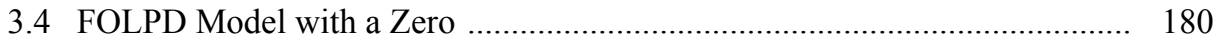

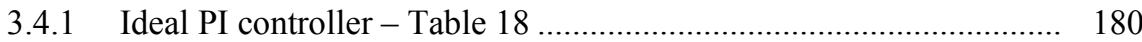

3.4.2 Ideal controller in series with a first order lag - Table 19 ................ 182

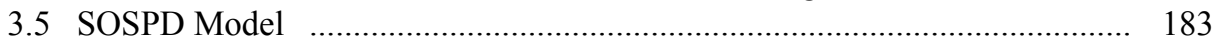

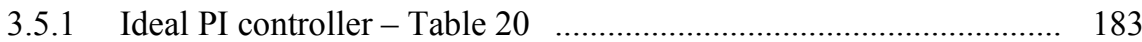

3.5.2 Ideal PID controller - Table 21 .................................................... 206

3.5.3 Ideal controller in series with a first order lag - Table $22 \ldots \ldots \ldots \ldots \ldots \ldots . . .232$

3.5.4 Controller with filtered derivative - Table 23................................. 236

3.5.5 Classical controller - Table 24 ................................................. 238

3.5.6 Generalised classical controller - Table 25 ................................... 251

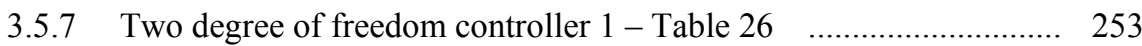

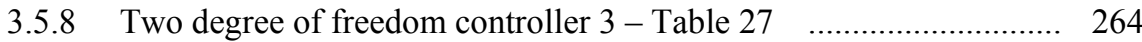

3.6 SOSPD Model with a Zero …………………………............................... 277

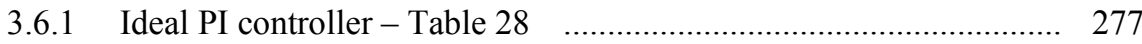

3.6.2 Ideal PID controller - Table 29 .................................................... 279

3.6.3 Ideal controller in series with a first order lag - Table $30 \quad \ldots \ldots \ldots \ldots \ldots . . .282$

3.6.4 Controller with filtered derivative - Table 31................................ 284

3.6.5 Classical controller - Table 32 ................................................... 286

3.6.6 Generalised classical controller - Table 33 ..................................... 288

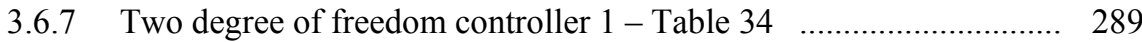

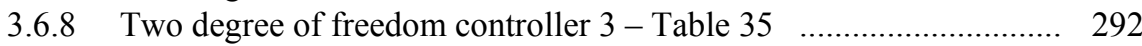

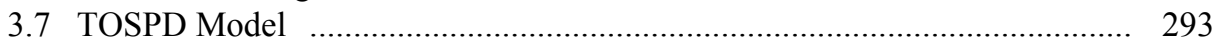

3.7.1 Ideal PI controller - Table 36 ................................................. 293

3.7.2 Ideal PID controller - Table 37 ...................................................... 296

3.7.3 Ideal controller in series with a first order lag - Table $38 \ldots \ldots \ldots \ldots \ldots \ldots . .297$

3.7.4 Controller with filtered derivative - Table 39 .................................. 298

3.7.5 Two degree of freedom controller 1 - Table 40 ................................ 299

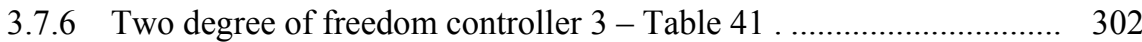

3.8 Fifth Order System Plus Delay Model ....................................................... 303

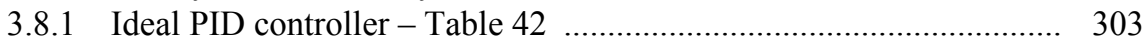

3.8.2 Controller with filtered derivative - Table 43 ................................. 305

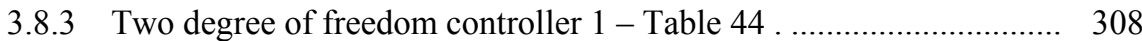

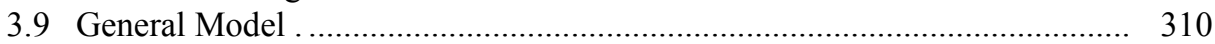

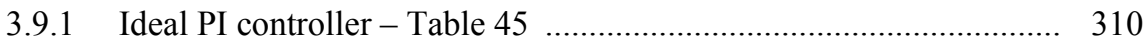

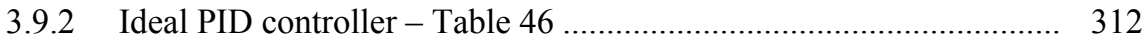

3.9.3 Ideal controller in series with a first order lag - Table $47 \ldots \ldots \ldots \ldots \ldots \ldots . . .315$

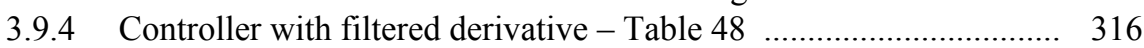

3.9.5 Two degree of freedom controller 1 - Table 49 _........................... 317

3.10 Non-Model Specific .......................................................................... 318

3.10.1 Ideal PI controller - Table 50 ....................................................... 318

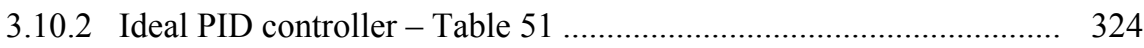

3.10.3 Ideal controller in series with a first order lag- Table 52 .............. 332

3.10.4 Controller with filtered derivative - Table 53 ............................... 336

3.10.5 Classical controller - Table 54 ................................................... 341

3.10.6 Generalised classical controller - Table 55 .................................... 343 
3.10.7 Two degree of freedom controller 1 - Table 56

3.10.8 Two degree of freedom controller 3 - Table 57

4. Controller Tuning Rules for Non-Self-Regulating Process Models ..................... 350

4.1 IPD Model ...................................................................................... 350

4.1.1 Ideal PI controller - Table 58 _.................................................... 350

4.1.2 Ideal PID controller - Table 59 ................................................... 359

4.1.3 Ideal controller in series with a first order lag - Table 60 .............. 364

4.1.4 Controller with filtered derivative - Table 61 .............................. 366

4.1.5 Classical controller - Table 62 ..................................................... 368

4.1.6 Generalised classical controller - Table 63 ................................... 371

4.1.7 Two degree of freedom controller 1 - Table 64 ........................... 372

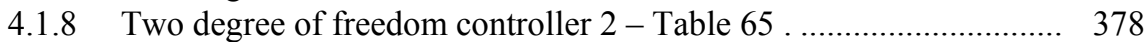

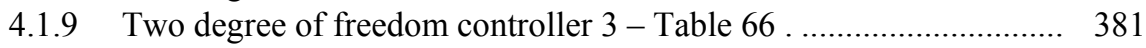

4.2 IPD Model with a Zero ............................................................................. 383

4.2.1 Ideal PI controller - Table 67 ..................................................... 383

4.3 FOLIPD Model .............................................................................. 385

4.3.1 Ideal PI controller - Table 68 ..................................................... 385

4.3.2 Ideal PID controller - Table 69 .................................................. 388

4.3.3 Ideal controller in series with a first order lag - Table 70 .............. 392

4.3.4 Controller with filtered derivative - Table 71 ............................... 394

4.3.5 Classical controller - Table 72 ...................................................... 395

4.3.6 Generalised classical controller - Table 73 ................................. 397

4.3.7 Two degree of freedom controller 1 - Table $74 \quad$............................ 399

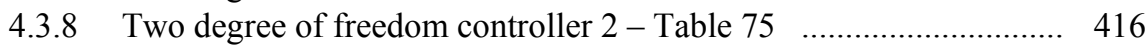

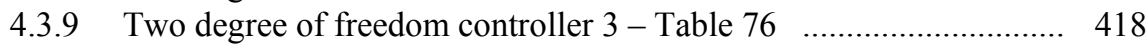

4.4 FOLIPD Model with a Zero …........................................................... 420

4.4.1 Ideal PID controller - Table 77 ................................................. 420

4.4.2 Ideal controller in series with a first order lag - Table $78 \quad \ldots \ldots \ldots \ldots \ldots . . . . .422$

4.4.3 Classical controller - Table 79 ..................................................... 423

$4.5 \mathrm{I}^{2} \mathrm{PD}$ Model .................................................................................... 424

4.5.1 Ideal PID controller - Table 80 _................................................. 424

4.5.2 Classical controller - Table 81 .................................................... 425

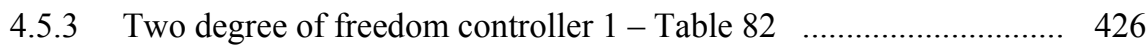

4.5.4 Two degree of freedom controller 2 - Table 83 ............................. 427

4.5.5 Two degree of freedom controller 3 - Table 84 ........................... 429

4.6 SOSIPD Model …...................................................................... 430

4.6.1 Ideal PI controller - Table 85 ..................................................... 430

4.6.2 Two degree of freedom controller 1 - Table 86 ........................... 431

4.7 SOSIPD Model with a Zero...................................................................... 436

4.7.1 Classical controller - Table 87 .................................................... 436

4.8 TOSIPD Model.................................................................................. 437

4.8.1 Two degree of freedom controller 1 - Table 88 ........................... 437 
4.9 General Model with Integrator ............................................................. 438

4.9.1 Ideal PI controller - Table 89 _...................................................... 438

4.9.2 Two degree of freedom controller 1 - Table 90 ............................ 439

4.10 Unstable FOLPD Model ....................................................................... 440

4.10.1 Ideal PI controller - Table 91 ..................................................... 440

4.10.2 Ideal PID controller - Table 92 .................................................... 447

4.10.3 Ideal controller in series with a first order lag - Table $93 \quad \ldots \ldots \ldots \ldots \ldots . . . .455$

4.10.4 Classical controller - Table 94 .................................................. 458

4.10.5 Generalised classical controller - Table 95 .................................. 462

4.10.6 Two degree of freedom controller 1 - Table 96 .............................. 463

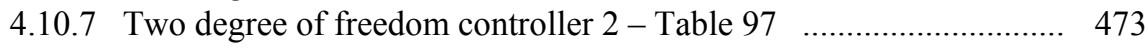

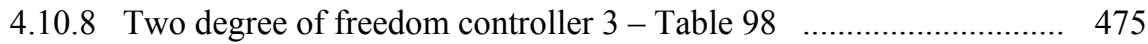

4.11 Unstable FOLPD Model with a Zero ...................................................... 480

4.11.1 Ideal PI controller - Table 99 .................................................... 480

4.11.2 Ideal controller in series with a first order lag - Table $100 \quad \ldots \ldots \ldots \ldots . . .481$

4.11.3 Generalised classical controller - Table 101 ................................. 483

4.11.4 Two degree of freedom controller 1 - Table 102 ......................... 484

4.12 Unstable SOSPD Model (one unstable pole) …......................................... 486

4.12.1 Ideal PI controller - Table 103 ..................................................... 486

4.12.2 Ideal PID controller - Table 104 f................................................ 488

4.12.3 Ideal controller in series with a first order lag - Table 105 ............ 490

4.12.4 Classical controller - Table 106 ................................................... 491

4.12.5 Two degree of freedom controller 1 - Table 107 ........................... 497

4.12.6 Two degree of freedom controller 3 - Table 108 .......................... 503

4.13 Unstable SOSPD Model (two unstable poles) ............................................ 506

4.13.1 Ideal PID controller - Table 109 .................................................... 506

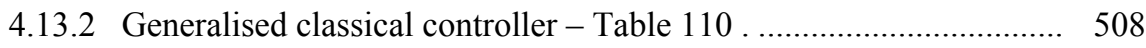

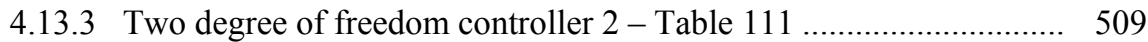

4.14 Unstable SOSPD Model with a Zero ........................................................ 511

4.14.1 Ideal PI controller - Table 112 .................................................... 511

4.14.2 Ideal controller in series with a first order lag - Table $113 \ldots \ldots \ldots \ldots . .513$

4.14.3 Generalised classical controller - Table 114 ............................... 516

4.14.4 Two degree of freedom controller 1 - Table 115 ........................... 518

4.14.5 Two degree of freedom controller 3 - Table $116 \ldots \ldots \ldots \ldots \ldots \ldots \ldots \ldots \ldots . . .520$

5. Performance and Robustness Issues in the Compensation of FOLPD

Processes with PI and PID Controllers ........................................................ 521

5.1 Introduction ...................................................................................... 521

5.2 The Analytical Determination of Gain and Phase Margin .......................... 522

5.2.1 PI tuning formulae .................................................................... 522

5.2.2 PID tuning formulae .......................................................... 525

5.3 The Analytical Determination of Maximum Sensitivity ............................ 529

5.4 Simulation Results ....................................................................... 529 
5.5 Design of Tuning Rules to Achieve Constant Gain and Phase Margins, for All Values of Delay

5.5.1 PI controller design.................................................................. 534

5.5.1.1 Processes modelled in FOLPD form................................. 534

5.5.1.2 Processes modelled in IPD form ........................................ 536

5.5.2 PID controller design ............................................................ 539

5.5.2.1 Processes modelled in FOLPD form - classical controller.... 539

5.5.2.2 Processes modelled in SOSPD form - series controller........ 541

5.5.2.3 Processes modelled in SOSPD form with a negative zero - classical controller ................................................. 542

5.5.3 PD controller design ........................................................ 542

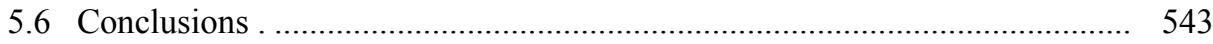

Appendix 1 Glossary of Symbols and Abbreviations ........................................ 544

Appendix 2 Some Further Details on Process Modelling ....................................... 551

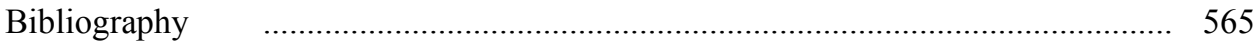

Index 